NASATM- TM66 O2

NASA Technical Memorandum 86655

Airborne Astronomy Program

Medium Altitude Missions Branch

Preprint Series 024

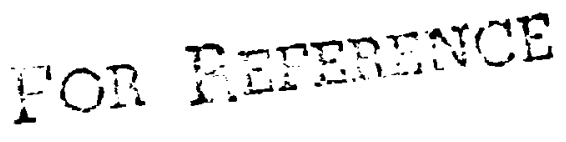

NASA-TM-86655

Cot TO NE TAKEN FBON THO BOOM

19850004527

\title{
An Infrared Study of the Bi-Polar Outflow Region GGD 12-15
}

Paul M. Harvey, Bruce A. Wilking, Marshall Joy, and Daniel F. Lester

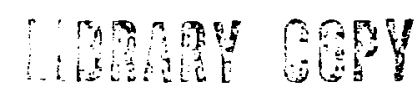

1904

WRELEY FESEARCH I EENTH LISTRBRY, NASA

HA:...TOS VIESNNAA

October 1984

N/SA

National Aeronautics and Space Administration

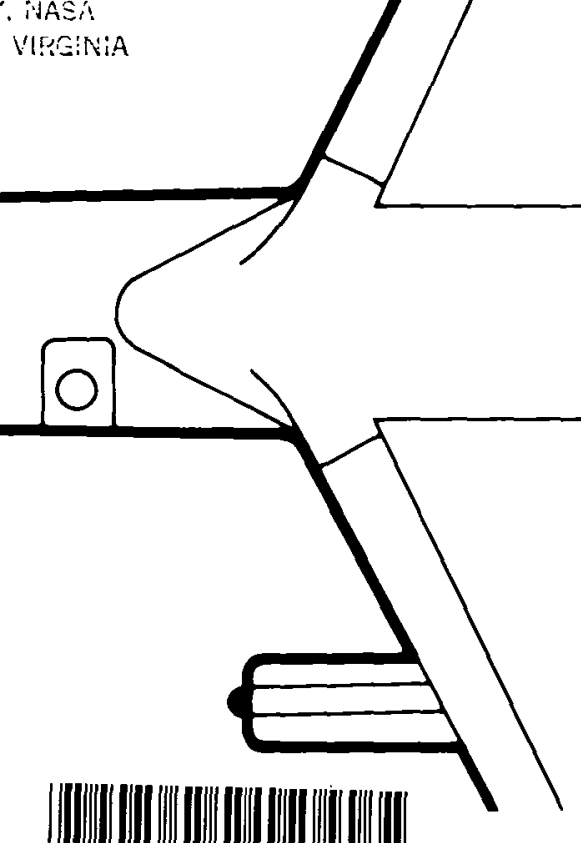




\section{An Infrared Study of the Bi-Polar Outflow Region GGD 12-15}

Paul M. Harvey

Bruce A. Wilking

Marshall Joy

Daniel F. Lester, University of Texas at Austin, Austin, Texas

\section{N/S}




\title{
An Infrared Study of the Bi-Polar \\ Outflow Region GGD 12-15
}

by

\author{
Paul M. Harvey, Bruce A. Wilking, Marshall Joy, and Daniel F. Lester \\ Astronomy Department \\ University of Texas at Austin \\ and \\ Visiting Astronomer at the Infrared Telescope Facility \\ which is operated by the University of Hawai i under contract \\ from the National Aeronautics and Space Administration.
}

Accepted (fiugust 1984) for publication in

THE ASTROPHYSICAI, JOURNAI,

$n^{\prime} 85=12835^{7}$ 
Infrared observations from 1-100 $\mu \mathrm{m}$ are presented for the region associated with a bipolar CO outflow source near the nebulous objects GGD 12-15. We find a luminous far-infrared source associated with a radio-continuum source in the area. This object appears to be a compact $H$ II region around a nearly mainsequence $B O$ star. We also find a faint $20 \mu \mathrm{m}$ source at the position of an $\mathrm{H}_{2} \mathrm{O}$ maser 30" northwest of the HII region. This object appears to be associated with but not coincident with a $2 \mu \mathrm{m}$ reflection nebula. We interpret this structure as evidence for a non-spherically symmetric, possibly disk-like dust distibution around the exciting star for the maser. This object probably powers the bi-polar co outflow although its Iuminosity is less than $10 \%$ that of the star which excites the compact HII region. A number of other $2 \mu \mathrm{m}$ sources found in the area are probably members of a recently formed cluster. 


\section{INTRODUCTION}

The region around GGD 12-15 (Gyulbudaghian, Glushkov and Denisyuk 1978) was identified as a likely site of recent star formation in the thorough radio wavelength study of Rodriguez et al. (1980) (RMHG). Subsequently, Rodriguez et al. (1982) found a bipolar outflow in ${ }^{12} \mathrm{CO}$ emission similar to a number of other regions discussed by Bally and Lada (1983). The general area in which the source of the outflow is centered contains a number of faint optical objects, a radio-continuum source, and an $\mathrm{H}_{2} \mathrm{O}$ maser 30 " away from the continuum source (RMHG). Reipurth and Wamsteker (1983) found several reddened near infrared sources in the region but were unable to identify unambiguously the sources of excitation of the maser and/or radio continuum source. At a distance of $1 \mathrm{kpc}$ (Racine and Van den Bergh 1970) this active region is only 1 pc in diameter.

In order to understand better the nature of the obscured stars in this region we undertook a variety of infrared observations at wavelengths from 1 to $100 \mu \mathrm{m}$. These data permit us to identify a group of embedded stars which probably represent a compact, newly-formed cluster in the region. The wide range of wavelengths observed permits us to estimate the total luminosities of the stars and to determine with much more certainty the sources of excitation of the maser and radio continuum source. The results of these observations show that the most luminous object, the exciting source for the radio continuum emission, appears to have a much smaller dynamical effect on the surrounding material than does the much lower luminosity exciting star associated with the maser. 


\section{OBSERVATIONS}

We observed the GGD 12-15 region in the near-infrared from 1-20 $\mu \mathrm{m}$ using the NASA IRTF on Mauna Kea in 1982 December, 1983 January, and 1984 February. We used standard facility photometers with the filters and aperture sizes listed in Table 1. Chopper spacings and direction were chosen to assure that "of $\mathrm{f}^{\text {" }}$ positions had surface brightnesses less than or equal to the lowest contour level shown in the maps below, typically 80" at $1-4 \mu \mathrm{m}$ and $50 "$ at $8-20 \mu \mathrm{m}$. The longer wavelength 50 and $100 \mu \mathrm{m}$ observations (Table 1) were made in 1983 October and 1984 February on NASA's Kuiper Airborne Observatory using the 3 spatial by 2 spectral element array photometer described by Wilking et al. (1984) with a 9' chopper throw roughly east-west. The 1-20 $\mu \mathrm{m}$ observations were calibrated relative to IRTF standard stars; the $50 / 100 \mu \mathrm{m}$ flux densities were calibrated relative to S140-IRS (Harvey, Wilking, and Joy 1984).

The IRTF observations consist of: (1) a $2.2 \mu \mathrm{m}$ map made with a 3.6 beam and $1 "$ spacing between samples (Figure 1), (2) a $20 \mu \mathrm{m}$ map of the brightest 20 $\mu \mathrm{m}$ emission region made with a 3.8 beam and 1 " spacing (Figure 4); (3) a small $20 \mathrm{um}$ map of the area near the $\mathrm{H}_{2} \mathrm{O}$ maser (Figure 2); (4) polarimetry of a nearinfrared source near the maser with a 3.6 beam (Table 3); and (5) photometry at several wavelengths between 1 and $20 \mu \mathrm{m}$ of a number of the sources found (Figure 3 and Tables 2 and 4). All IRTF positions were of set from SAO $\# 132924$ and are believed accurate to $\pm 1 "$ absolute. Relative positions of nearby sources are probably accurate to $\pm 0.5^{\prime \prime}$. The KAO observations consist of maps at 50 and 100 $\mu \mathrm{m}$ made with $30^{\prime \prime}$ beams and $20^{\prime \prime}$ sample spacing over a $5^{\prime} \times 5^{\prime}$ area and with $5^{\prime \prime}$ spacing in the central $3^{\prime} \times 3^{\prime}$ as shown in Figure 5 . The positions are believed accurate to $\pm 7^{\prime \prime}$. 


\section{III, RESULTS}

\section{a) The Maser Region}

Figure 1 shows a local $2.2 \mu \mathrm{m}$ flux maximum (Source 9E) about 4" east and slightly south of the $\mathrm{H}_{2} \mathrm{O}$ maser position (RMHG). The small area, $20 \mu \mathrm{m}$ map of this region (Figure 2), however, shows a clear $20 \mu \mathrm{m}$ flux maximum at the maser position (Source 9Ni) within the relative positional uncertainties. There is little or no $20 \mu \mathrm{m}$ emission from the nearby $2 \mu \mathrm{m}$ peak position. This morphology is similar to that seen in NGC $6334-V$ by Harvey and Wilking (1984). They proposed that such structure was consistent with a picture in which the $20 \mu m$ source/ $\mathrm{H}_{2} \mathrm{O}$ maser represents the position of a single, obscured exciting star which is surrounded by a dense dust cloud with non-spherically symmetric geometry. In such a picture the $2 \mu \mathrm{m}$ emission to the southeast of the GGD 12-15 $20 \mu \mathrm{m} /$ maser source represents scattered light from the central source which emerges along a line of lower extinction than our line of sight to the central source.

This picture is consistent with two additional observations shown in Figure 3 and Table 3. Figure 3 shows the results of HKL photometry at a number of points along a line parallel to the co outflow through the maser position. These data show that the near-infrared "nebulosity" becomes progressively bluer from a point 2" east of the maser to $6 "$ " east as would be expected for reflection nebulosity viewed through decreasing amounts of extinction. The K-L color measured at the maser position is not as red as at the point 2 " east, but this could be due to contamination of the $K$ measurement by the very extended $2.2 \mu \mathrm{m}$ emission discussed in Sec. III-d if that emission is relatively blue. Table 3 shows the results of polarimetric measurements made at the position of peak $2.2 \mu \mathrm{m}$ surface brightness southeast of the maser position. The detection of 
significant polarization with the $E$ vector along a $N-S$ line is consistent with the interpretation of this source as reflected light from an embedded object to the west, 1.e. possibly the maser $/ 20 \mu \mathrm{m}$ exciting star.

Elgure 3 also shows that there is a second, redder and weaker near-infrared source 12" northwest of the maser which is colinear with the maser and the southeastern $2 \mu \mathrm{m}$ peak. This might represent the northwestern lobe of a bipolar reflection nebula analogous to that seen in NGC 6334-V (Harvey and Wilking 1984), if it were on the far side of the exciting star and viewed through a higher column density of material. In that case the circumstellar dust cloud might have a disk-like geometry as proposed for NGC 6334-V (Harvey and Wilking $1984)$.

An upper limit to the total luminosity of the exciting star for the maser $/ 20 \mu \mathrm{m}$ source may be estimated from the shape of the low-level 50 and $100 \mu \mathrm{m}$ contours at the position of the maser relative to the point source response as shown in the map of Figure 5. This, together with a lower limit estimated from the 1-20 $\mu \mathrm{m}$ photometry imply a total luminosity of $10^{3} \mathrm{~L}_{\odot} \geq \mathrm{L} \geq 200 \mathrm{~L} \odot$. A zeroage main-sequence star of luminosity $5 \times 10^{2} \mathrm{~L}_{\odot}$ would have a spectral type of $B 5$. Its total $2.2 \mu \mathrm{m}$ brightness would be $K-9.5 \mathrm{mag}$. Since the integrated $2.2 \mu \mathrm{m}$ brightness in the supposed bi-polar reflection nebula is only $K-11.0$ mag., a B5 ZAMS star could just power the observed reflection nebulae if the reflection efficiency were reasonably high. If the central star has a $2 \mu \mathrm{m}$ excess as appears commonly in many other young objects (see sec. III-d), then the required reflection efficiency and extinction requirements are quite reasonable. 


\section{b) The Radio Continuum Source}

The peak 20-100 $\mu \mathrm{m}$ emission in the GGD $12-15$ region is coincident with the position of the $6 \mathrm{~cm}$ radio continuum source (RMHG) as shown in Figures 4 and 5 . Therefore, since virtually all the luminosity of this region is radiated in the infrared, this object is the dominant luminosity source. The total infrared luminosity in a $30^{\prime \prime}(0.15 \mathrm{pc})$ diameter area centered on the $6 \mathrm{~cm}$ source is L $8.0 \pm 2 \times 10^{3} \mathrm{~L}_{\odot} . \quad$ RMHG estimated that a $10^{4} \mathrm{~L}_{\odot}, \mathrm{B} 0.5$ zero-age main-sequence star could produce the $H$ II region inferred from the $6 \mathrm{~cm}$ flux. Since that luminosity is nearly equal to the observed infrared luminosity, this object appears to be simply a highly obscured, young H II region around a main-sequence or nearly main-sequence star.

The 8-20 $\mu \mathrm{m}$ energy distribution of the core of this object (Table 4) indicates a relatively $\operatorname{cool}\left[\mathrm{T}_{\mathrm{C}}(8.7-12.5 \mu \mathrm{m})-150 \mathrm{~K}\right]$ dust temperature and silicate optical depth $\tau_{9.7 \mu m} \geq 1.0$. Using a ratio of $A_{v} / \tau_{\text {sil }}-17$ (Rieke and Lebofsky 1984), this implies $A_{v} \geq 17 \mathrm{mag}$. There is no obvious compact feature in the $2.2 \mu \mathrm{m}$ map (Figure 1) at the position of the $20 \mu \mathrm{m}$ peak to a level of K-14.0 mag, although there is some low-level diffuse emission. A ZAMS BO.5 star would have an apparent $K$ magnitude of 7.8 mag if there were no obscuration. Therefore the observed lack of a point-like exciting source for the H II region implies an extinction at $2.2 \mu \mathrm{m} \mathrm{A}_{\mathrm{K}} \geq 6 \mathrm{mag}$, suggesting an even larger $\mathrm{A}_{\mathrm{V}} \geq 55$ mag. Another limit may be placed on the extinction to the $H$ II region by comparing the expected $2 \mu \mathrm{m}$ free-free emission from the $\mathrm{H}$ II region to the observed $2 \mu \mathrm{m}$ flux. From the observed radio flux of $120 \mathrm{mJy}$ (RMHG) we expect $\mathrm{K}$ $10.3 \mathrm{mag}$ due to free-free, bound-free, and line emission. This implies $\mathrm{A}_{\mathrm{v}} \geq 40$ mag. 


\section{c) The Other $\underline{2} \underline{\mu m}$ Sources}

There are ten other local flux maxima in Figure 1 above the level of 2.4 $\mathrm{mJy} /$ beam ( $K S 13.5$ ) in addition to the source associated with the maser (IRS 9 ). Sources $3,5,6$, and 10 appear essentially pointlike relative to our 3.5 beam down to the $25 \%$ contour or below. Sources 1,2 , and possibly 8 may be double at the $25-50 \%$ contour level with component separations of $2 "-4 "$. Sources 7,11 , and 12 are somewhat broader than a point source below the 50\% contour. These may be either point sources superimposed on a diffuse background or genuinely resolved sources.

Although some or all of these sources could represent reflection nebulae excited by the star which excites the $H$ II region or by other obscured luminosity sources, it seems more likely that at least the point-like $2 \mu \mathrm{m}$ sources probably represent the positions of discrete stellar objects. This is because, first, a highly obscured star which is luminous enough to produce a bright, compact reflection nebula would also be likely to manifest itself as a nearby, detectable longer wavelength source. Secondly, if many of the $2 \mu \mathrm{m}$ sources were reflection nebulae powered by the H II region source, the total observed $2 \mu \mathrm{m}$ flux density in the region and reasonable reflection efficiencies would require that the $H$ II exciting star have a strong $2 \mu \mathrm{m}$ excess. Although the stellar atmospheres of highly obscured young stars have not been wellstudied, this seems unlikely for a star whose total luminosity and radioinferred Lyman continuum luminosity agree so well at a spectral type near Bo.5. The best method to answer this question with certainty in the future is probably a combination of polarimetric mapping of the entire region and spectroscopy of the individual sources. 
Based on the above arguments, we tentatively interpret the eastern peak of source 1, and sources $3,5,6$, and 10 as obscured stars because of the pointlike contours within a couple of arcseconds of their centers. Sources $7,8,11$, and 12 are probably individual stars, but could be $2 \mu \mathrm{m}$ reflection or emission nebulae illuminated by their own obscured exciting stars or by the exciting star for the obscured H II region. Source 2 appears nearly unresolved along one axis and may represent either such a neblilosity or a partially resolved double star. On the basis of the 1-20 $\mu \mathrm{m}$ photometry and the lack of more than one local flux maximum in the $50 / 100 \mu \mathrm{m}$ maps none of these sources can have a luminosity significantly greater than $10^{3} \mathrm{~L}_{\odot}$. Table 2 lists the extinction, $A_{V}$, derived by assuming that the $\mathrm{J}$ and $\mathrm{H}$ flux densities represent emission from the reddened photosphere of a not, star-like object. A ratio of $A_{V} / E_{J-H}=11$ was used (Becklin et al. 1978). The de-reddened absolute $\mathrm{K}$ magnitudes are also listed in Table 2. For many of the sources these magnitudes are too bright to represent emission from the photospheres of ZAMS stars within the luminosity limit $L \leq 10^{3} \mathrm{~L}_{\odot}$. This appears to be a common property of pre-main-sequence stars (Lada and Wilking 1984; Harvey, Wilking, and Joy 1984); the excess may be due to circumstellar gas or dust emission or photospheres which are cooler than appropriate for the zero-age-main-sequence.

d) Large Scale Structure - The Diffuse $2 \mu \mathrm{m}$ and $100 \mu \mathrm{m}$ Radiation Both the $2.2 \mu \mathrm{m}$ and $100 \mu \mathrm{m}$ maps show evidence for diffuse emission over much of the mapped area. At $100 \mu \mathrm{m}$ this emission is probably due to dust within the extended molecular cloud which is heated both centrally by the H II region and by fainter nearby stars. A visible reflection nebula ' 1 ' southwest is probably responsible for the extended contours in that direction. Because of the strong concentration of the $50 \mu \mathrm{m}$ emission around the $\mathrm{H}$ II region, it 
appears that the visible reflection nebula probably represents only a minor total source of Iuminosity.

The diffuse $2 \mu \mathrm{m}$ emission is unlikely to be due to thermal dust emission from typical interstellar dust since dust temperatures above $10^{3} \mathrm{~K}$ would be implied. Two other possibilities are: (1) reflected emission from the central exciting star for the H II region and possibly some of the other sources, or (2) anomalous thermal emission similar to that found by Sellgren, Werner, and Dinerstein (1983) near visible reflection nebulae, due possibly to nonequilibrium heating of very small grains, or (3) the combined background of a large number of fainter stars. The total diffuse $2.2 \mu \mathrm{m}$ emission over the mapped area amounts to $-50 \mathrm{mJy}(\mathrm{K}-10 \mathrm{mag})$. This could be supplied by the central star of the $H$ II region if of order $10 \%$ of the $2 \mu \mathrm{m}$ luminosity could be converted into reflection nebulosity. This would imply a highly anisotropic distribution of dust around the central star since the extinction along the line of sight is greater than $5 \mathrm{mag}$ at $2.2 \mu \mathrm{m}$. Without further observations it will be difficult to determine the source of this diffuse emission with certainty.

\section{DISCUSSION}

a) The Source of the co outflow

This region provides an interesting comparison between two young objects, (1) a compact H II region/far-infrared source, and (2) a much lower luminosity $\mathrm{H}_{2} \mathrm{O}$ maser/IR source. Our infrared data show, in fact, that the luminosity of the object associated with the $\mathrm{H}_{2} \mathrm{O}$ maser is at least a factor of 8 less than that of the exciting star for the H II region. Three pieces of evidence suggest, however, that the $C O$ outflow in this region is associated with the 
maser source rather than with the H II region. First, for outflow sources in general (Bally and Lada 1983) there is a very strong statistical correlation between the presence of an outflow and the presence of an $\mathrm{H}_{2} \mathrm{O}$ maser. Second, geometrically the $\mathrm{H}_{2} \mathrm{O}$ maser/IR source in the GGD $12-15$ area is significantly closer to the center of the outflow than is the $H$ II region/far-ir source (Rodriguez et al. 1982). Third, the agreement in alignment between the CO outflow axis and the displacement of the $2 \mu \mathrm{m}$ source IRS $9 \mathrm{E}$ from the $20 \mu \mathrm{m}$ source IRS $9 M$ at the maser position suggests a physical relationship between the infrared source/maser and the larger scale CO outflow. Taken together, these three arguments provide strong circumstantial evidence that the maser source powers the outflow. Higher angular resolution radio observations of the region near the maser are probably needed to be absolutely certain of this conclusion.

Rodriguez et al. (1982) and Bally and Lada (1983) have argued that the bipolar outflow occurs in a large fraction of young stars at some time in their evolution. If that is true, and if the absence of an observable outflow associated with the compact $H$ II region is real and not simply due to an effect such as inauspicious orientation, this suggests that its exciting star has not yet passed through the outflow stage or has already completed it. Since the $H$ II region is resolved ( $\mathrm{RMHG}$ ) it seems more likely that its exciting star has evolved beyond the outflow stage since most outflow sources of similar Iuminosity have unresolved or non-existent $H$ II regions (Bally and Lada 1983; Wynn-Williams 1982). This result is consistent with the idea that the star which excites the H II region and that which excites the maser formed coevally and the more luminous star, which excites the H II region, evolved more rapidly. 


\section{b) The Assoclated Infrared Cluster}

There are at least $f$ ive and perhaps ten more $2 \mu \mathrm{m}$ sources within the mapped area (Figure 1) which probably represent obscured stars. This cluster is thus comparable in density of stars to that associated with the bi-polar infrared nebula NGC 6334-V-IRS 4 (Harvey and Wilking 1984) at equal luminosity limits. Unlike that cluster, however, the most luminous young star is not the object associated with a high velocity outflow.

As noted earlier, the de-reddened absolute $K$ magnitudes of virtually all the sources are too bright to be due to photospheric emission from ZAMS stars with low enough luminosities to be consistent with the far-infrared observations. This suggests that most of these stars are young and possibly pre-main-sequence. It is interesting then that there appears to be only a single "outflow" source in this cluster. This is consistent with the idea (Bally and Lada 1983, and references therein) that the outflow stage is a fairly short lived, transient event in the lives of young stars. 


\section{Acknowledgements}

We thank the ground and flight crew of the Kuiper Observatory and the staff of the Infrared Telescope Facility for their assistance with these observations and for their efforts which provided such excellent telescope performance. This research was supported in part by NASA grant NAG 2-67. 


\section{References}

Bally, J., and Lada, C.J. 1983, Ap. J., 2265, 824.

Becklin, E.E., Matthews, K., Neugebauer, G., and Willner, S.P. 1978, Ap. J., 220,831

Gillett, F.C., Forrest, W.F., Merrill, K.M., Capps, R.W., and Soifer, B.T. 1975, Ap. J., 200, 609 .

Gyulbudaghian, A.L., Glushkov, Yu. I., and Denisyuk, E.K. 1978, Ap. J.

(Letters), 224, L137.

Harvey, P.M., and Wilking, B.A. 1984, Ap. ‥ (Letters), in press.

Harvey, P.M., Wilking, B.A., and Joy, M. 1984, Nature, in press.

Racine, R., and Van den Bergh, S. 1970, in The Spiral Structure of Our Galaxy,

ed. W. Becker and G. Contopoulos (Dordrecht: Reidel).

Reipurth, B., and Wamsteker, W. 1983, Astron. Ap., 119, 14.

Rieke, G.H., and Lebofsky, M.J. 1984, preprint.

Rodriguez, L.F., Carral, P., Ho, P.T.P., and Moran, J.M. 1982, Ap. J., 260, 635.

Rodriguez, L.F., Moran, J.M., Ho. P.T.P., and Gottlieb, E.W. 1980, Ap. J., 235,

845.

Sellgren, K., Werner, M.W., and Dinerstein, H.L. 1983, Ap. J. (Letters), 271,

L13.

Wilking, B.A., Harvey, P.M., Lada, C.J., Joy, M., and Doering, C.R. 1984, Ap. J., , in press.

Wynn-Williams, C.G. 1982, Annual Reviews of Astronomy and Astrophysics, 20, 587. 
TABLE 1

Instrumental Parameters

\begin{tabular}{|c|c|c|c|}
\hline Filter & $\lambda_{\text {eff }}(\mu \mathrm{m})$ & Beam Size & Telescope \\
\hline$J$ & 1.25 & $3.6^{\prime \prime}$ & IRTF \\
\hline $\mathrm{H}$ & 1.65 & $"$ & $"$ \\
\hline K & 2.2 & $"$ & $"$ \\
\hline L & 3.5 & $"$ & $"$ \\
\hline 8.7 & 8.7 & $3.8 "$ & $"$ \\
\hline 9.7 & 9.7 & $"$ & $"$ \\
\hline $\mathrm{N}$ & 10.2 & $"$ & $"$ \\
\hline 10.3 & 10.3 & $"$ & $"$ \\
\hline 12.6 & 12.6 & $"$ & $"$ \\
\hline$Q$ & 20.0 & $"$ & $"$ \\
\hline 50 & 47 & 27 & $\mathrm{KAO}$ \\
\hline 100 & 95 & 29 & $"$ \\
\hline
\end{tabular}


TABLE 2

1-20 $\mu \mathrm{m}$ Photometry

\begin{tabular}{|c|c|c|c|c|c|c|c|c|c|c|}
\hline$\underset{\#}{\text { Source }}$ & $\begin{array}{l}\text { R.A. }(1950) \\
06^{\mathrm{h}} 08^{\mathrm{m}}\end{array}$ & $\begin{array}{l}\text { Dec. } \\
-06^{\circ}\end{array}$ & $\begin{array}{c}\mathrm{K} \\
\text { mag. }\end{array}$ & $\mathrm{J}-\mathrm{H}$ & $\mathrm{H}-\mathrm{K}$ & $\mathrm{K}-\mathrm{L}$ & $\mathrm{N}$ & $Q$ & $\begin{array}{l}\text { Derived } \\
\text { Av }\end{array}$ & $M_{k}$ \\
\hline 1 & 24.01 & $11 ' 21: 5$ & 11.57 & 1.11 & 0.68 & 0.62 & $6.9 \pm .25$ & $2.7 \pm .28$ & 20 & 0.2 \\
\hline 2 & 23.84 & $11^{\prime} 15^{\prime \prime}$ & 11.01 & 1.65 & 1.57 & 2.02 & 2.58 & -1.47 & 18 & -1.0 \\
\hline 3 & 24.25 & $11^{\prime} 12$ & 12.04 & 1.75 & 1.16 & 1.23 & 4.72 & -0.86 & 19 & $-0.1^{\circ}$ \\
\hline 4 & 23.98 & $11^{\prime} 07^{\prime \prime}$ & 14.28 & $1.3 \pm 0.2$ & 1.14 & 3.24 & 2.46 & -2.82 & 14 & 2.7 \\
\hline 5 & 23.35 & $11^{\prime} 02: .5$ & 11.40 & 1.29 & 1.11 & 1.14 & $6.2 \pm .25$ & $2.8 \pm .25$ & 14 & -0.2 \\
\hline 6 & 22.96 & $10^{\prime} 59^{\prime \prime}$ & 11.68 & $\geq 3$ & 2.34 & 1.89 & $6.2 \pm .25$ & $3.2 \pm .3$ & 33 & -2.0 \\
\hline 7 & 24.31 & $10^{\prime} 57^{\prime \prime}$ & 12.19 & $2.7 \pm 0.2$ & 2.23 & 1.80 & & $\geq 3.0$ & 30 & -1.1 \\
\hline 8 & 24.09 & $10^{\prime} 53^{\prime \prime}$ & 12.43 & 2.35 & 1.83 & 1.36 & & $\geq 2.8$ & 26 & -0.4 \\
\hline $9 \mathrm{E}$ & 25.95 & $10^{\prime} 51^{\prime \prime}$ & 12.03 & 1.98 & 1.12 & 1.11 & $6.6 \pm .3$ & $\geq 3.0$ & 30 & -1.5 \\
\hline $9 \mathrm{M}$ & 25.75 & $10^{\prime} 49.5$ & 14.40 & $\geq 1.0$ & 1.89 & 1.3 & $5.9 \pm .25$ & 1.85 & & \\
\hline 10 & 23.96 & $10^{\prime} 36: 5$ & 9.57 & 2.45 & 1.97 & 1.50 & $5.6 \pm .2$ & 23.0 & 27 & -3.4 \\
\hline 11 & 25.10 & $10 ' 53 . " 0$ & 13.51 & 2.5 & 1.76 & 1.32 & $>6.5$ & $>2.0$ & 27 & 0.5 \\
\hline 12 & 22.75 & $10146: 7$ & 12.15 & 1.63 & 0.61 & 0.48 & & & 18 & 0.2 \\
\hline
\end{tabular}

Observational uncertainties are $\pm 0.1 \mathrm{mag}$ at $\mathrm{J}, \mathrm{H}, \mathrm{K}, \mathrm{L}, \pm 0.15 \mathrm{mag}$ at $\mathrm{N}$, and $\pm 0.2 \mathrm{mag}$ at $\mathrm{Q}$ unless otherwise noted. 
TABLE 3

Polarimetry of IRS 9-SE

P

$\theta$
$\mathrm{H}(1.65 \mu \mathrm{m})$

$4.8 \pm 1.0 \%$

$170^{\circ} \pm 15$
$K(2.2 \mu \mathrm{m})$

$2.5 \pm 0.5 \%$

$178^{\circ} \pm 12$ 
TABLE 4

\section{Narrow-Band $10 \mu \mathrm{m}$ Photometry}

$\begin{array}{ccc}\begin{array}{c}\text { Wavel ength } \\ (\mu \mathrm{m})\end{array} & \begin{array}{l}\text { IRS 2 } \\ (\mathrm{Jy})\end{array} & \begin{array}{c}\text { IRS } 4 \\ (\mathrm{Jy})\end{array} \\ 8.7 & 2.27 & 1.36 \\ 9.7 & 2.53 & 1.21 \\ 10.3 & 3.31 & 2.33 \\ 11.6 & 5.94 & 7.71 \\ 12.5 & 7.92 & 12.9 \\ 20 \text { (broad) } & 39 & 134\end{array}$

Uncertainties as in Table 2 . 


\section{Figure Captions}

Figure 1 - Map of the GGD $12-15$ area at $2.2 \mu \mathrm{m}$ made with 3.6 beam (FWHM). Contours are at levels $1,1.5,2,4,8,16,32,64$, and $128 \times 0.6 \mathrm{mJy}$ per beam. The total region which was mapped includes most of the area shown, with the exception of small areas in the northeast and northwest corners. The various sources found are labeled with the numbers used in Table 2 . The dashed square around IRS-9M indicates the area of the $20 \mu \mathrm{m}$ map shown in Figure 2. Likewise for the dashed rectangle around IRS 1-4 and Eigure 4. The dashed line through IRS $-9 M$ and $9 E$ shows the scan line along which the observations in Figure 3 were made. The "H" and surrounding dashed circle show the position and size of the compact $H$ II region found by RMHG which is also coincident with IRS 4 of Table 2. In this map and those that follow, the lowest contour is at a level where the signalto-noise ratio was of order $3: 1$.

Figure 2 - Map with a 3.8 beam at $20 \mu \mathrm{m}$ centered on the position of the $\mathrm{H}_{2} \mathrm{O}$ maser (RMHG) indicated by the dashed cross near the center. Fluxes are in Jy per beam. Contours are sketched at $0.5,1.0$ and $1.5 \mathrm{Jy}$ per beam.

Figure 3 - Scans at $1.65,2.2$, and $3.5 \mu \mathrm{m}$ made through maser position $(0,0)$ along Co outflow axis with a 3.6 beam. Offsets shown are R.A. and Dec $(+$ east, + north $)$ in arcseconds.

Figure 4 - Map made with 3.8 beam at $20 \mu \mathrm{m}$ of the brightest $20 \mu \mathrm{m}$ sources found in the GGD 12-15 region, IRS $-2,3,4$. Contours are at levels 10, 20, $40,60,80,100,120$, and $135 \mathrm{Jy}$ per beam. The dashed circle and plus sign Indicate the size and central position of the $H$ II region found by 
RMHG.

Figure 5 - Maps made at 50 and $100 \mu \mathrm{m}$ with 28" beams. Contours are at levels $90,80,60,50,40,20,10,5$, and $(100 \mu \mathrm{m}$ only) $2.5 \%$ of the peak fluxes fluxes which were $2.2 \times 10^{3}$ and $2.1 \times 10^{3} \mathrm{Jy}$ per beam respectively at 50 and $100 \mu \mathrm{m}$. The plus sign indicates the position of the compact $\mathrm{H}$ II region and the cross shows the position of the $\mathrm{H}_{2} \mathrm{O}$ maser ( $\mathrm{RMHG}$ ). 
P. M. Harvey, M. Joy, D. F. Lester, and B. A. Wilking Astronomy Department

University of Texas

Austin, TX 78712 


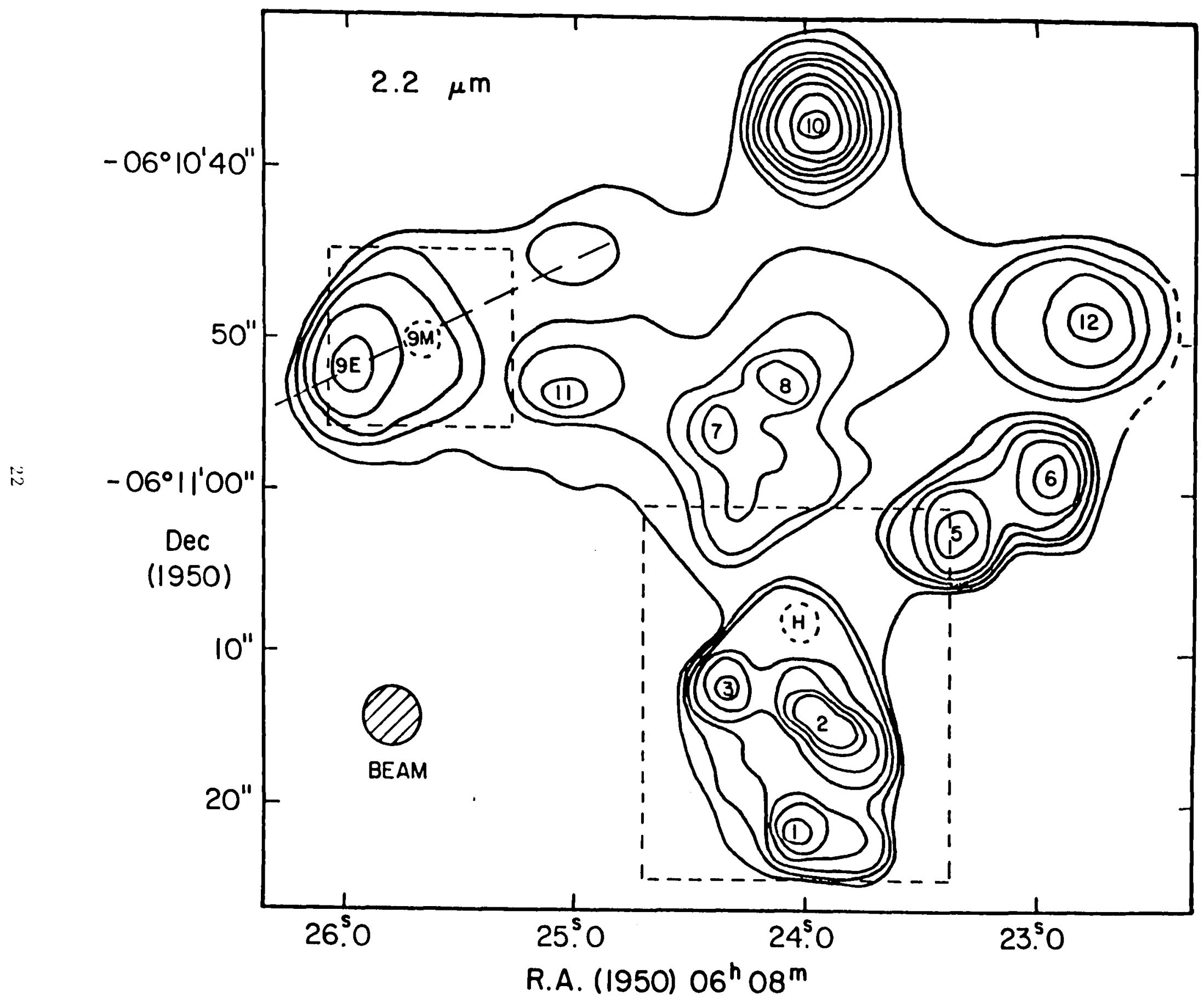


w

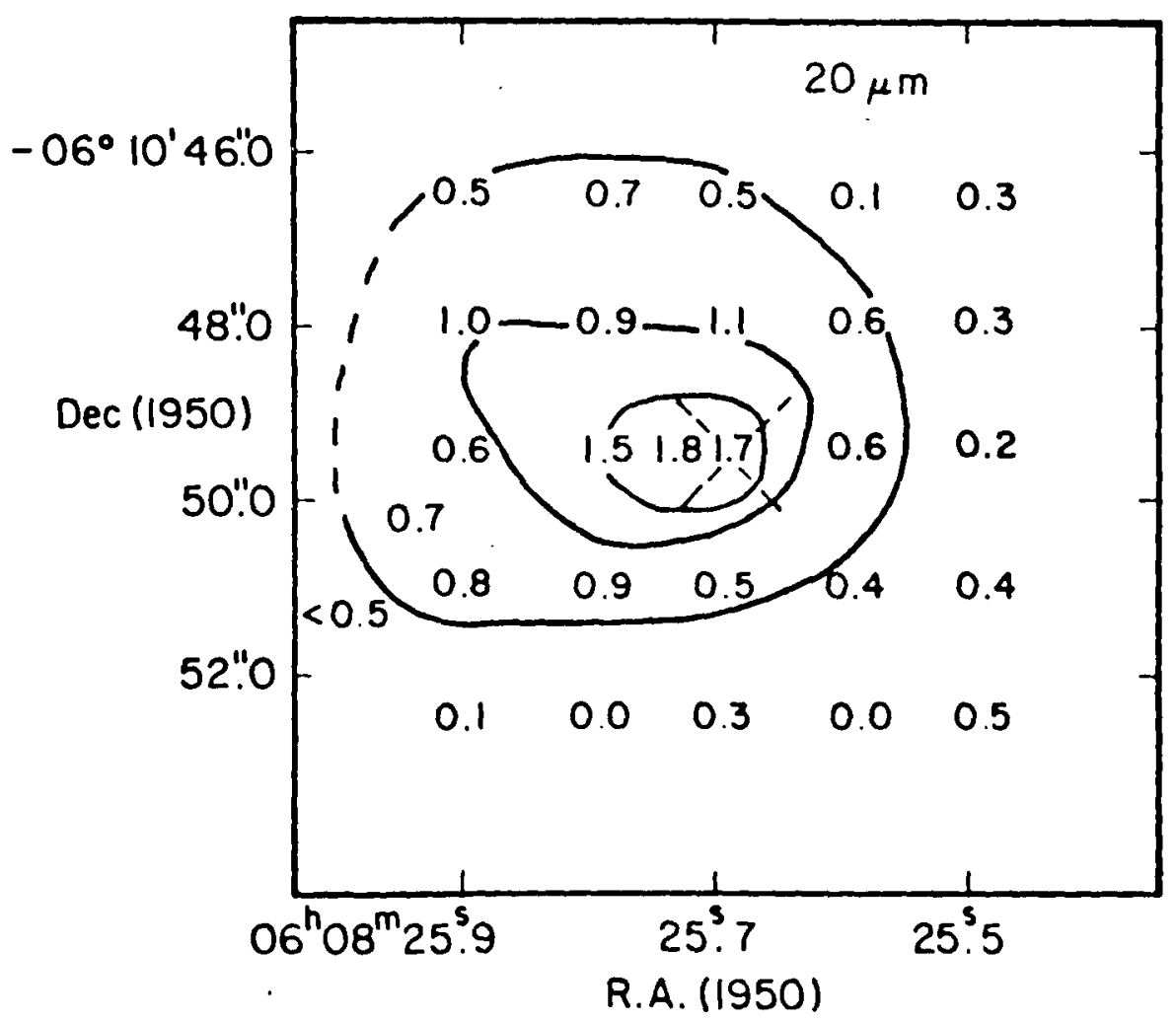

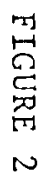




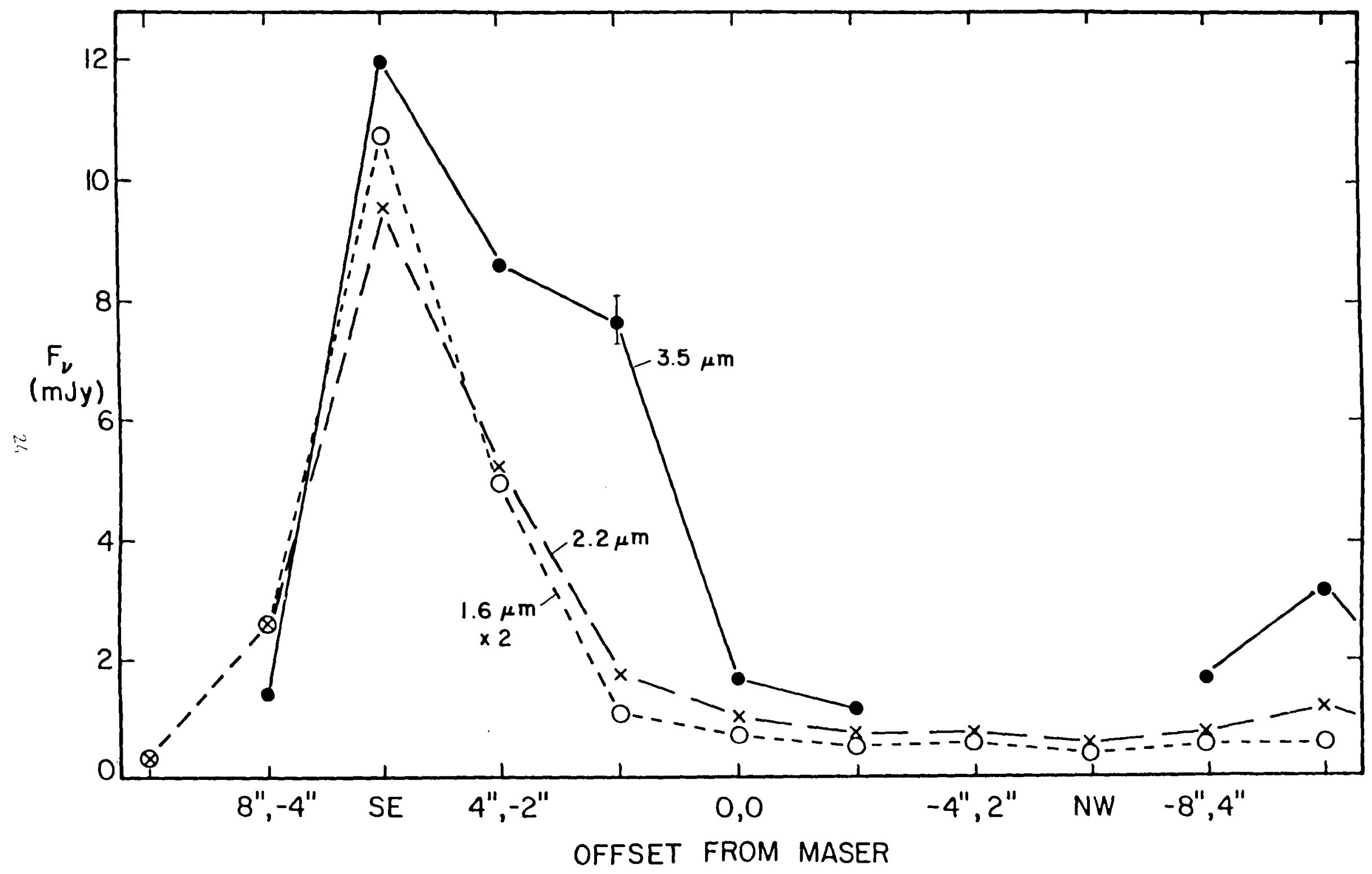

FIGURE 3 
FIGURE 4

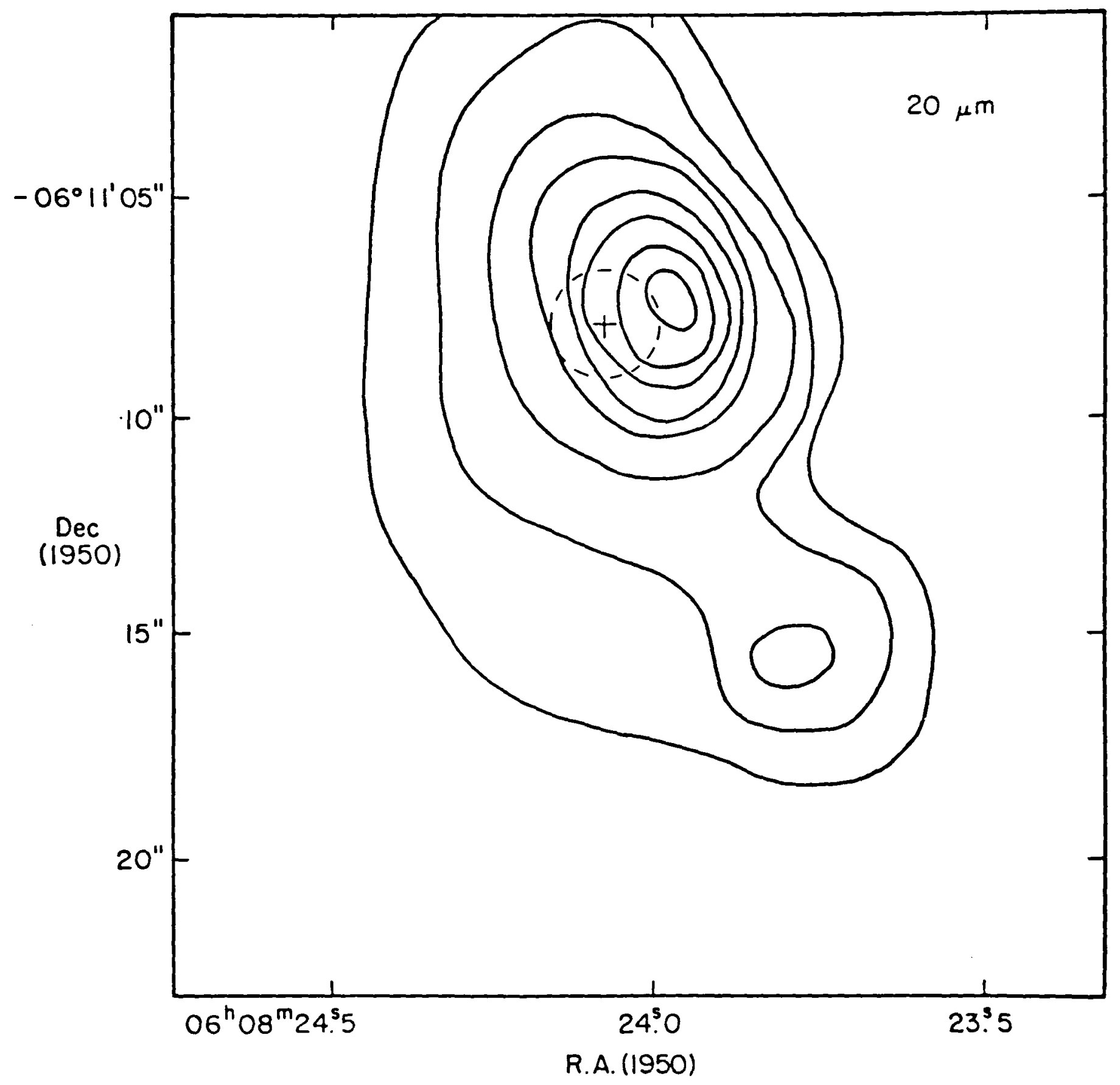




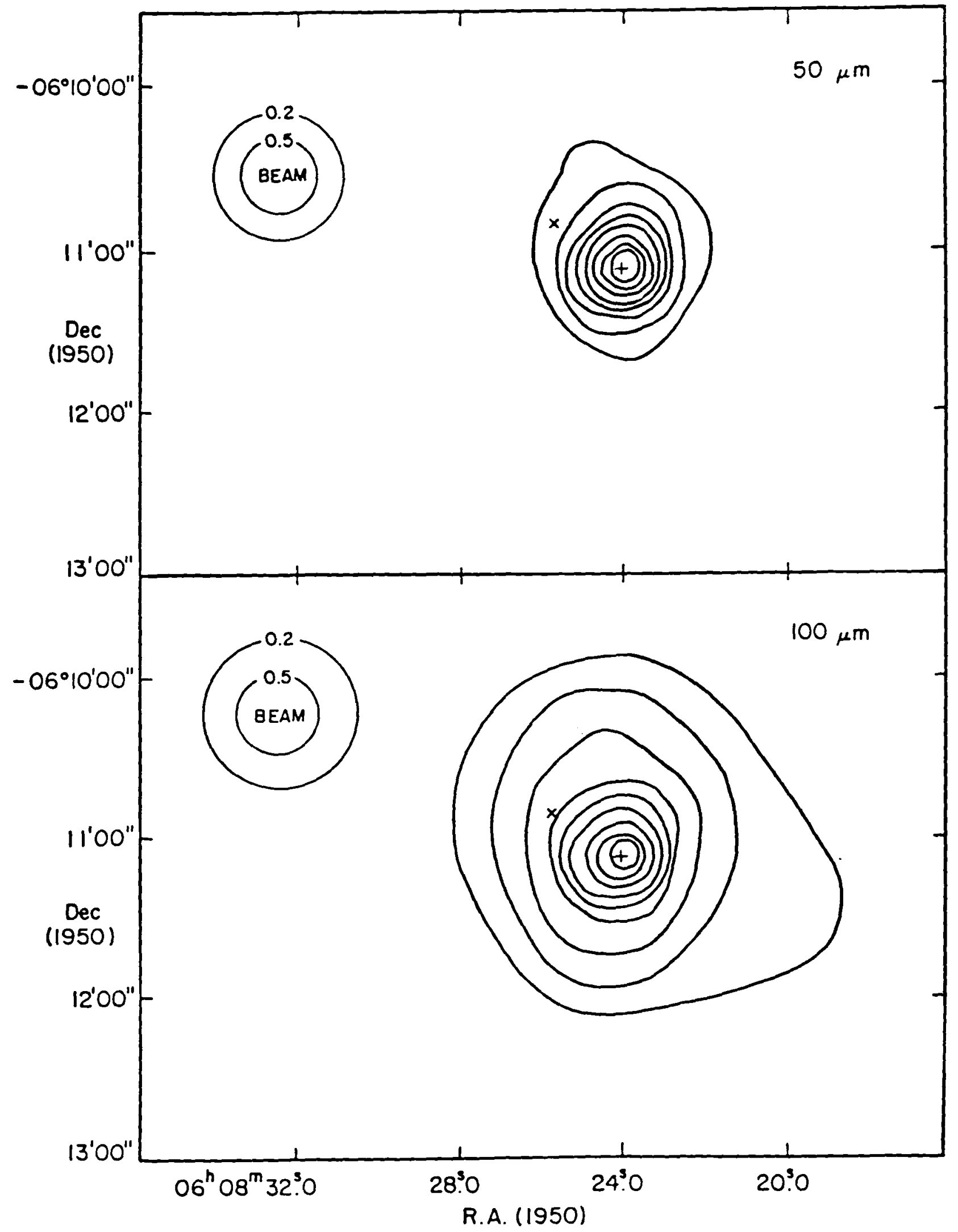




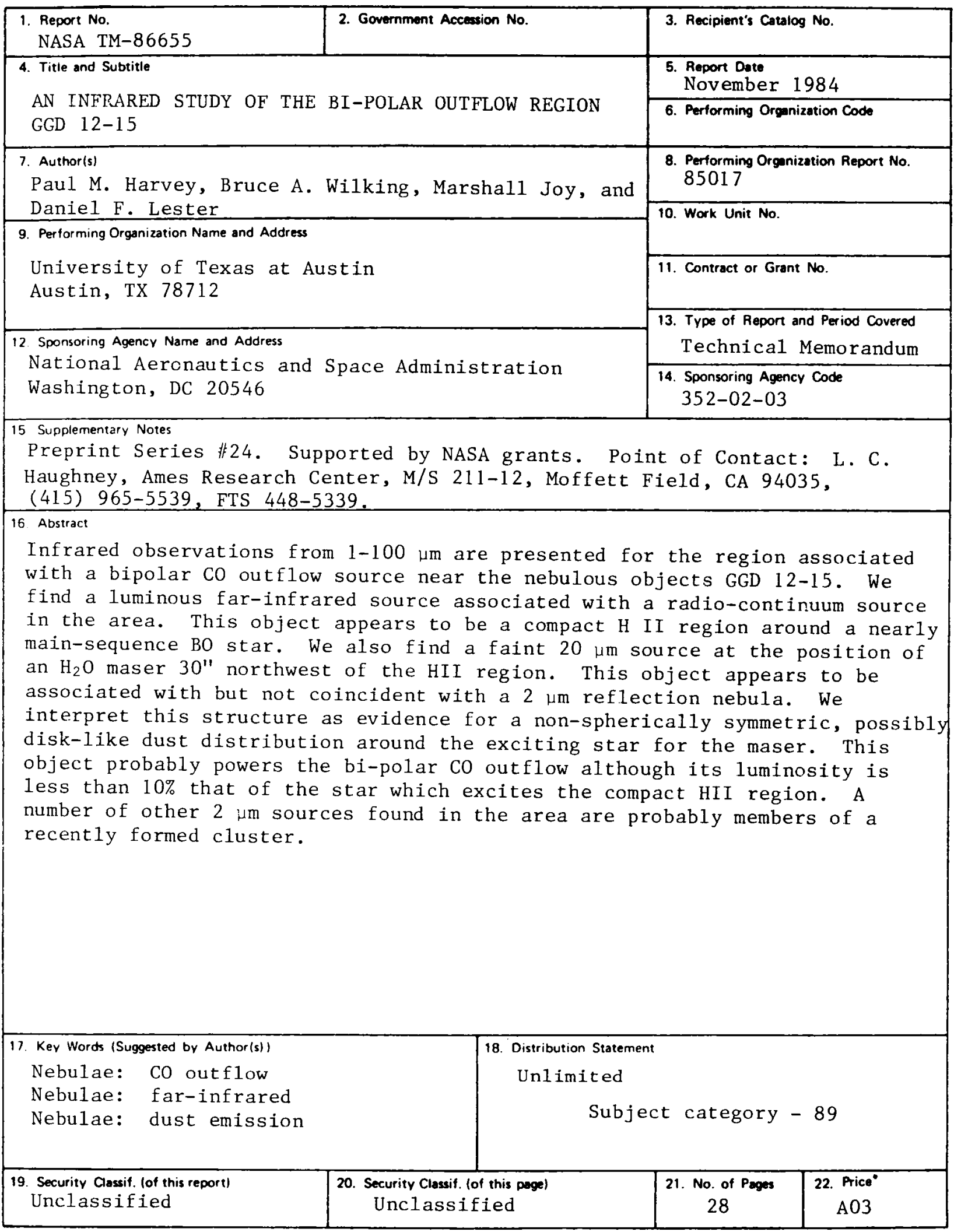


End of Document 